\title{
Resistência Natural de Eucalyptus urophylla e Corymbia citriodora à Coptotermes gestroi (Isoptera; Rhinotermitidae) em Laboratório
}

\author{
Rodrigo Bastos Rodrigues ${ }^{1}$, Edvá Oliveira Brito ${ }^{2}$ \\ ${ }^{1}$ Universidade Federal Rural do Rio de Janeiro - UFRRJ \\ ${ }^{2}$ Departamento de Produtos Florestais, Universidade Federal Rural do Rio de Janeiro -UFRRJ
}

\begin{abstract}
RESUMO
Este trabalho teve como objetivo avaliar a resistência natural da madeira de Eucalyptus urophylla e Corymbia citriodora ao ataque de Coptotermes gestroi. A avaliação da resistência a cupins foi realizada segundo a norma ASTM D - 3345 (American..., 1994). A média de perda de massa do Pinus elliottii foi superior à do Eucalyptus urophylla e Corymbia citriodora, indicando maior susceptibilidade à ação de Coptotermes gestroi. A superior mortalidade dos cupins, observada em Eucalyptus urophylla e Corymbia citriodora, sugere que essas madeiras possuem características mais deletérias à Coptotermes gestroi, em relação às de Pinus elliottii.
\end{abstract}

Palavras-chave: perda de massa, mortalidade, deterioração.

\section{Natural Resistance of Eucalyptus urophylla and Corymbia citriodora to Coptotermes gestroi (Isoptera; Rhinotermitidae)}

\begin{abstract}
This work aimed to evaluate the natural resistance of Eucalyptus urophylla and Corymbia citriodora to Coptotermes gestroi attacks. The termites' resistance evaluation was performed according to the ASTM D - 3345 (American..., 1994) standard. The average weight loss of Pinus elliottii was greater than that of Eucalyptus urophylla and Corymbia citriodora; this indicates that Pinus elliottii has a greater susceptibility to Coptotermes gestroi attacks. The mortality of termites showed that E. urophylla and C. citriodora wood is less attractive and more resistant to degradation by Coptotermes gestroi, while P. elliottii is more vulnerable to attacks.
\end{abstract}

Keywords: mass loss, mortality, deterioration.

\section{INTRODUÇÃO}

A madeira é um produto largamente utilizado em todo o mundo para construção de prédios, móveis, dormentes de estradas de ferro, pontes e uma série de outras utilidades não menos importantes (Gomes et al., 2005).

Um dos fatores que limitam a utilização da madeira para suas diversas finalidades é relativo à sua durabilidade e conhecimento ao ataque de xilófagos, principalmente dos cupins, o que se torna um requisito muito importante, para a utilização correta da madeira, principalmente nas indústrias de movelaria e construção civil (Oliveira, 2006).

Segundo Paes (2002), a resistência natural da madeira à deterioração é a capacidade inerente 
da madeira de uma espécie florestal de resistir à ação de agentes deterioradores, principalmente os agentes biológicos. Essa característica varia entre e nas espécies, havendo, assim, registros de grandes diferenças entre a resistência da madeira de árvores de uma mesma espécie e, inclusive, dentro do mesmo indivíduo (Panshin \& De Zeeuw, 1980 apud Lima, 1997).

Dessa forma, o conhecimento da resistência natural da madeira é de suma importância para a recomendação de seu emprego mais adequado, evitando-se gastos desnecessários com a reposição de peças e reduzindo os impactos sobre as florestas remanescentes, uma vez que o emprego da madeira mais apropriada às condições de risco de deterioração reduz o corte de árvores (Paes et al., 2004).

Diante de informações desse tipo, determinada madeira poderá ter preferência em relação às demais quanto maior for a sua durabilidade natural, pois se evitariam os inconvenientes advindos da utilização de produtos químicos, alguns bastante tóxicos, empregados no tratamento de madeiras de baixa durabilidade, a fim de lhes conferir um desempenho satisfatório em serviço (Oliveira, 1997).

Diversos são os autores que relatam a ação de substâncias químicas como terpenoides, terpenos, quinonas, polifenóis e outros extrativos como repelentes e/ou substâncias tóxicas aos cupins ou aos seus simbiontes. A mortalidade destes organismos poderia ser ocasionada pela ação direta sobre os cupins ou do desequilíbrio causado sobre seus simbiontes. A quantidade desses micro-organismos presentes no intestino dos cupins pode se tornar insuficiente para digerir a celulose, influenciando o hábito alimentar desses insetos, o que poderia causar-lhes a morte (Supriana, 1985 apud Abreu \& Silva, 2000).

Os cupins são insetos sociais que vivem em colônias cujos indivíduos são divididos em castas com funções definidas: reprodução, trabalho e defesa da colônia. De modo geral, as espécies que atacam a madeira podem ser divididas em subterrâneas e de madeira seca. Os cupins são, dentre os insetos, os mais severos agentes destruidores da madeira (Paes \& Vital, 2000), e os de solos, ou subterrâneos, são responsáveis pelos maiores volumes de perdas de madeira no mundo (Hunt \& Garratt, 1967; Richardson, 1993).

O objetivo deste trabalho foi avaliar a resistência natural de Eucalyptus urophylla S.T.Blake, Corymbia citriodora (Hook) K. D. Hill \& L. A. S. Johnson e Pinus elliottii Engelm como testemunha, à ação de Coptotermes gestroi.

\section{MATERIAL E MÉTODOS}

\subsection{Amostragem}

Para estudo da resistência natural das madeiras, foram amostradas árvores de Eucalyptus urophylla $\left(0,70 \mathrm{~g} / \mathrm{cm}^{3}\right)$ e Corymbia citriodora $\left(0,85 \mathrm{~g} / \mathrm{cm}^{3}\right)$, com idades de 21 anos, provenientes de plantios existentes na Floresta Nacional Mário Xavier (FLONA), situada no $\mathrm{Km} 50$ do município de Seropédica, RJ. As espécies analisadas abrangeram um total de quatro árvores (2 por espécie), com diâmetro médio de $30 \mathrm{~cm}$ e $29 \mathrm{~m}$ de comprimento.

Uma árvore de Pinus elliottii $\left(0,58 \mathrm{~g} / \mathrm{cm}^{3}\right)$, com idade de 21 anos, medindo $28 \mathrm{~m}$ de comprimento e diâmetro médio de $26 \mathrm{~cm}$, foi abatida no Instituto de Florestas da UFRRJ, e sua madeira forneceu amostras para serem utilizadas como testemunha.

\subsection{Análise acelerada de laboratório}

\subsubsection{Preparação dos corpos de prova}

Para confecção dos corpos de prova (Figura 1), foi empregada somente a base das árvores utilizadas, contendo $50 \mathrm{~cm}$ de comprimento. Cada base foi descascada e desdobrada em sarrafos. Os sarrafos, por sua vez, foram transformados em corpos de prova de 2,54 x 2,54 x 0,64 cm, e constituídos de cerne somente. Assim, as dimensões dos corpos de prova, seguiram a descrição da norma ASTM D 3345 (American..., 1994), que padroniza o teste de resistência de madeiras ao ataque de cupins subterrâneos, em condições de laboratório. Os corpos de prova foram secos em estufa $105 \pm 2{ }^{\circ} \mathrm{C}$ por 24 horas e foi determinada a massa de cada amostra, como recomendado pela ASTM D-1413 (American..., 1994) e os valores foram utilizados no cálculo da perda de massa sofrida pelos corpos de prova submetidos aos cupins. 


\subsubsection{Coleta de cupins}

Os cupins foram coletados de uma colônia localizada no Instituto de Florestas da Universidade Federal Rural do Rio de Janeiro, utilizando iscas de madeira como atrativo. As iscas foram levadas para o laboratório de Preservação de Madeira do Departamento de Produtos Florestais - IF da UFRRJ e armazenadas em uma caixa para seleção dos insetos mais sadios.

Para isso, foram utilizadas iscas de papelão (cortadas em formato quadrangular com cerca de $20 \times 20 \mathrm{~cm}$ ) que foram acomodadas no fundo da caixa e umedecidas com água destilada. Esperou-se um prazo de 3 dias para que os cupins saíssem da madeira e se dispusessem no papelão. Após esse prazo, os papelóes foram desmontados sobre uma bandeja

A seguir, os cupins foram transportados para um balde de $10 \mathrm{~L}$ contendo 20 toalhas de papel, sendo 10 úmidas (com água destilada), ligeiramente amassadas, e 10 secas.

Após 6 horas, os cupins se acomodaram por entre as toalhas, ficando os mais fracos e danificados por cima das toalhas e os mais vigorosos direcionados para as toalhas úmidas. As toalhas secas foram retiradas e as úmidas levadas para o laboratório para a coleta dos cupins, o que foi feito sobre uma bandeja.
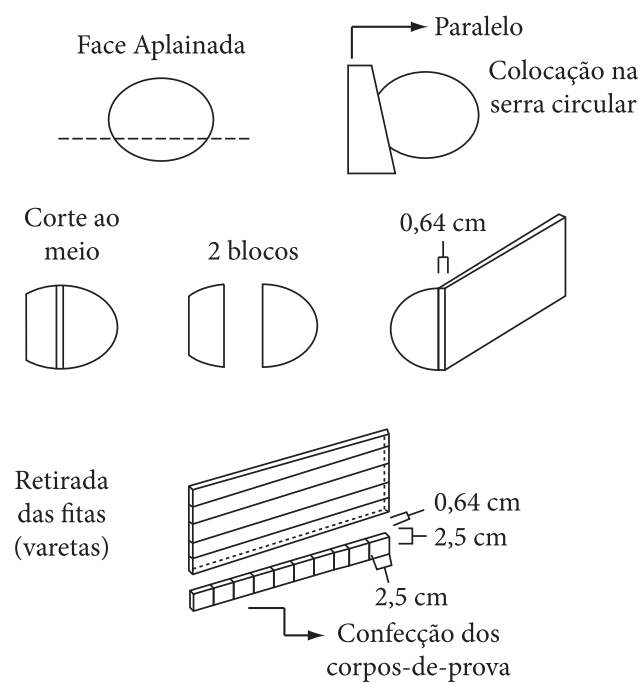

Figura 1. Esquema de confecção dos corpos de prova (Brito, 2011).

Figure 1. Retreat scheme of samples. (Brito, 2011).

\subsubsection{A montagem do experimento foi realizada da seguinte forma}

Em vidros lavados e secos em estufa $\left(100{ }^{\circ} \mathrm{C}\right)$ foram adicionados $200 \mathrm{~g}$ de areia e $39 \mathrm{~mL}$ de água destilada (quantidade alcançada segundo a fórmula de saturação). Após a adição da água, os vidros permaneceram em repouso durante 12 horas. Após esse período, um corpo de prova foi acomodado em cada vidro, sendo este encaixado na sua lateral e enterrado na areia de forma que a face do corpo de prova ficasse visível para análise, na lateral do recipiente. Para cada madeira foram feitas dez repetições (cinco frascos com areia, bloco de madeira e amostras de cupins). O mesmo foi feito com a testemunha.

\subsubsection{Cálculo do ponto de saturação de água na areia}

Em um béquer, posicionado sobre uma balança de precisão, foram adicionados $100 \mathrm{~g}$ de areia seca em estufa $\left(100^{\circ} \mathrm{C}\right)$ e água destilada.

O ponto de saturação é definido quando a adição de água resultar em sobra de água na superfície da areia.

$\%$ de saturação $=($ peso da água $/$ peso da areia $) \times 100$

$$
\% \text { de água adicional }=\% \text { de saturação }-7
$$

Foi pesado $1 \pm 0,05 \mathrm{~g}$ de exemplares de Coptotermes gestroi e adicionado em cada frasco. Cuidou-se para que $90 \%$ dos cupins utilizados fossem operárias, pois são os indivíduos desta casta que são responsáveis pelos danos na madeira. Após a adição dos cupins, os frascos foram tampados com tela fina como recomendado por Paes (1997).

Além disso, foram utilizados cinco vidros sem bloco (somente com água, areia e cupim) para avaliar a vitalidade dos cupins. Os vidros foram mantidos entre 25,5 e $27^{\circ} \mathrm{C}$ de temperatura durante quatro semanas. Foram pesados aleatoriamente cinco vidros. Os vidros foram examinados no final da primeira e da quarta semana, identificando túneis e a posição em geral dos cupins no vidro. Semanalmente, os cinco vidros, examinados aleatoriamente, foram pesados, adicionando-se mais água se a umidade tivesse caído por volta de $2 \%$ ou mais. Após uma semana, os vidros sem blocos foram analisados para 
verificação da mortalidade dos cupins. Após quatro semanas, os vidros foram desmontados, removendose e limpando-se os blocos.

O ensaio de resistência natural da madeira a cupins xilófagos com as amostras obtidas dos toretes de $50 \mathrm{~cm}$ de espessura foi executado segundo as recomendações da norma ASTM D-3345 (American..., 1994), com alguns dos ajustes recomendados por Paes (1997).

Sendo assim, para avaliar a resistência da madeira de E. urophylla, C. citriodora e P. elliottii, foram computados a perda de massa (cálculo segundo a fórmula abaixo), o desgaste (Tabela 1) e a mortalidade dos cupins, avaliada ao término do experimento (Tabela 2).

A perda de massa dos corpos de prova (secos em estufa $105 \pm 2{ }^{\circ} \mathrm{C}$ por 24 horas) foi avaliada com tomadas de peso antes e após o término do ensaio, segundo a Equação 1:

$$
\mathrm{pm}=\frac{\text { massa inicial }- \text { massa final }}{\text { massa inicial }}
$$

\subsection{Análise estatística}

Para análise dos dados da perda de massa, foi utilizado o programa estatístico SAEG (Sistema para Análises Estatísticas). Foi realizado o teste de Lilliefors para verificar a normalidade dos dados, como não apresentaram, foram transformados em porcentagem e, após transformação, aplicou-se o Teste de Tukey para análise de variâncias, ao nível de $5 \%$ de significância.

\section{RESULTADOS E DISCUSSÃO}

Avaliando-se a resistência dos corpos de prova em relação à mortalidade dos cupins, observou-

Tabela 1. Classificação do material conforme o tipo de desgaste, segundo a norma ASTM D-3345 (1994).

Table 1. Classes of resistance based on natural decay, according to the ASTM D-3345 standard (1994).

\begin{tabular}{lc}
\multicolumn{1}{c}{ Tipos de desgaste } & Nota \\
\hline Sadio, permitindo escarificações superficiais. & 10 \\
Ataque superficial & 9 \\
Ataque moderado, havendo penetração & 7 \\
Ataque intensivo & 4 \\
Falha, havendo ruptura dos corpos de prova & 0 \\
\hline
\end{tabular}

se que a madeira de eucalipto proporcionou taxas de mortalidade superiores às registradas em pinus (Tabela 3), sendo, portanto, sua resistência classificada como alta em relação à ação de C. gestroi, segundo os parâmetros expressos na Tabela 2. Variáveis como a densidade e a presença de extrativos poderiam ter atuado como barreiras físicas e químicas aos cupins. Sendo assim, diversos autores relatam que esses extrativos agem como repelentes e/ou substâncias tóxicas aos cupins ou aos seus simbiontes (Abreu \& Silva, 2000).

A madeira de pinus teve a resistência classificada como moderada, provavelmente por possuir menor efeito dos extrativos, e também uma menor densidade em relação a E. urophylla e C. citriodora, características que podem ter facilitado a ação dos cupins.

No entanto, para explicar o fato da madeira de $P$. elliottii ter sido classificada com resistência moderada, em relação à mortalidade dos cupins, o que, em se tratando de madeira de pinus entende-se como uma classificação não habitual, pode-se, então, especular que a espécie "elliottii", que apresenta altos teores de extrativos, em comparação com outras espécies de pinus (Brito, 2011), tenha sua classificação influenciada por essa característica. Soma-se a isso a idade da árvore utilizada para retirar as amostras, sendo de 21 anos, podendo, dessa forma, ter uma porção de cerne maior que uma árvore mais jovem. Nesse sentido, o cerne é habitualmente mais denso, o que também poderia ter influenciado a ação dos cupins.

Ainda, a densidade apresentada pela madeira de Pinus elliottii pode ser considerada alta quando comparada com outras espécies de Pinus ou

Tabela 2. Avaliação da resistência a cupins pela porcentagem de mortalidade, segundo a norma ASTM D-3345 (American..., 1994).

Table 2 . Resistance evaluation by mortality percentage of termites, according to the ASTM D-3345 standard (American..., 1994).

\begin{tabular}{|cc|}
\hline Avaliação da resistência & Mortalidade (\%) \\
\hline Baixa & $0-33$ \\
\hline Moderada & $34-66$ \\
\hline Alta & $67-99$ \\
\hline Total & 100 \\
\hline
\end{tabular}


Tabela 3. Percentual de mortalidade dos cupins após 30 dias de exposição aos corpos de prova de madeira de E. urophylla, C. citriodora e P. elliottii à ação de C. gestroi em ensaio de laboratório, Seropédica, RJ, 2010.

Table 3. Termite mortality after 30 days of exposure to body-of-proof timber E. urophylla, C. citriodora and P. elliottii to the action of C. gestroi in laboratory test. Seropédica, RJ, 2010.

\begin{tabular}{|c|c|c|c|c|c|}
\hline \multicolumn{2}{|c|}{ E. citriodora } & \multicolumn{2}{|c|}{ E. urophylla } & \multicolumn{2}{|c|}{ Pinus elliottii } \\
\hline Amostra & $\%$ & Amostra & $\%$ & Amostra & $\%$ \\
\hline 1 & 90 & 1 & 80 & 1 & 50 \\
\hline 2 & 80 & 2 & 90 & 2 & 50 \\
\hline 3 & 100 & 3 & 80 & 3 & 60 \\
\hline 4 & 100 & 4 & 90 & 4 & 53 \\
\hline 5 & 80 & 5 & 90 & 5 & 57 \\
\hline 6 & 90 & 6 & 90 & 6 & 60 \\
\hline 7 & 90 & 7 & 90 & 7 & 60 \\
\hline 8 & 90 & 8 & 80 & 8 & 80 \\
\hline 9 & 90 & 9 & 100 & $\mathrm{X}$ & $\mathrm{X}$ \\
\hline 10 & 100 & 10 & 90 & $\mathrm{X}$ & $\mathrm{X}$ \\
\hline Média & 81 & & 88 & & 58 \\
\hline
\end{tabular}

$\mathrm{X}$ : As respectivas amostras foram perdidas devido à proliferação de outros organismos xilófagos.

mesmo com a mesma espécie, com idades menores. Assim sendo, a idade da árvore tem influência nas variações de certas propriedades da madeira, como o comprimento dos traqueoides, a densidade, a percentagem de lenho tardio e o ângulo microfibrilar. Conforme Zobel (1992), qualquer alteração no padrão de crescimento de uma árvore, resultante ou não de intervenções silviculturais, pode resultar em variações na qualidade da madeira. Nesse sentido, o registro de uma resistência moderada, conferido pela observação da mortalidade dos cupins, na madeira de pinus, pode ter explicações frente às peculiaridades da madeira e da espécie de pinus utilizada nesta pesquisa.

Nos recipientes sem madeira, a longevidade dos cupins foi constatada com sua sobrevida por um período maior do que nos recipientes com madeira. Fato esse que sugere que qualquer uma das madeiras avaliadas neste trabalho pode ter características que promovam mortalidade à C. gestroi.

De acordo com Paes et al. (2003), o tempo para a morte dos cupins é um indicativo da resistência natural da madeira.

A perda de massa média dos corpos de prova confeccionados com madeira de E. urophylla e C. citriodora demonstrou-se diferente quando comparada pelo Teste de Tukey a 5\% de significância com o valor médio registrado nas observações realizadas nos corpos de prova de madeira de P. elliottii (Tabela 4).
A média de perda de massa do P. elliottii foi superior à do E. urophylla e C. citriodora, indicando maior susceptibilidade à ação de C. gestroi.

Analisando o desgaste sofrido, percebeu-se que os corpos de prova confeccionados com madeira de pinus e que foram utilizados como testemunhas sofreram danos mais intensos em relação às outras madeiras avaliadas, apresentando perfurações e não somente escarificações superficiais, o que, além da maior perda de massa, também demonstra superior susceptibilidade ao desgaste ocasionado pela ação de C. gestroi (Tabela 5).

Para a madeira de E. urophylla e C. citriodora, observaram-se escarificações superficiais, não havendo ruptura ou penetração.

Paes \& Vital (2000) relatam que a madeira de E. urophylla é altamente vulnerável ao ataque de cupins, embora neste ensaio tenha apresentado boa resistência natural à ação de C. gestroi.

Para o P. elliottii, observou-se que os danos nos corpos de prova foram mais evidentes em comparação com os confeccionados com as outras madeiras avaliadas neste ensaio, corroborando com resultados de estudos anteriores que caracterizaram este tipo de material como tendo baixa resistência quando em contato com cupins (Pêgas, 2007). Ainda, segundo Foelkel (2011), a madeira dos Pinus é considerada de baixa resistência aos ataques de cupins, pela sua baixa densidade e composição química. Embora neste experimental a madeira de 
Tabela 4. Perda de massa média dos corpos de prova de madeira de E. urophylla, C. citriodora e P. elliottii expostos à ação de C. gestroi em ambiente de laboratório, Seropédica, RJ, 2010.

Table 4. Average mass loss of body-of-proof timber E. urophylla, C. citriodora and P. elliottii, exposed to the action of $C$. gestroi in a laboratory environment. Seropédica, RJ, 2010.

\begin{tabular}{|cc|}
\hline Madeira & Perda de massa média (\%) \\
\hline P. elliottii & $3,85^{\mathrm{a}}$ \\
\hline C. citriodora & $2,89^{\mathrm{b}}$ \\
\hline E. urophylla & $2,86^{\mathrm{b}}$ \\
\hline
\end{tabular}

Médias seguidas de mesma letra não diferem entre si, pelo Teste de Tukey a $5 \%$ de significância.

Tabela 5. Desgaste sofrido pelos corpos de prova segundo o sistema nota descrição ASTM D-3345 (American..., 1994), Seropédica, RJ, 2010.

Table 5. Decay of samples according description note system ASTM D-3345 (American..., 1994). Seropédica, RJ, 2010.

\begin{tabular}{|cccc|}
\hline \multirow{4}{*}{ Amostra Nota } \\
\cline { 2 - 4 } & C. citriodora & E. urophylla & P. elliottii \\
\hline 1 & 9 & 7 & 7 \\
2 & 7 & 9 & 7 \\
3 & 10 & 7 & 4 \\
\hline 4 & 10 & 9 & 7 \\
\hline 5 & 7 & 9 & 7 \\
\hline 6 & 9 & 9 & 4 \\
\hline 7 & 9 & 9 & 4 \\
\hline 8 & 9 & 7 & 7 \\
\hline 9 & 9 & 10 & $\mathrm{X}$ \\
\hline 10 & 10 & 9 & $\mathrm{X}$ \\
\hline $\begin{array}{c}\text { Desgaste } \\
\text { médio }\end{array}$ & 8,9 & 8,5 & 5,8 \\
\hline $\begin{array}{l}\text { X: As respectivas amostras } \\
\text { proliferação de outros organismos xilófagos. }\end{array}$ & \\
\hline
\end{tabular}

P. elliottii tenha sido classificada com resistência moderada, frente à mortalidade de C. gestroi.

Santos et al. (2010), ao utilizarem iscas armadilha de pinus em áreas com a presença de C. gestroi, relataram que houve total consumo das amostras antes do período predeterminado para as suas coletas, sugerindo que a partir do momento que os cupins da espécie C. gestroi localizam a fonte de alimento, o consumo é intensificado até o seu fim. Em condições de laboratório, com a quantidade de operárias utilizadas ( $1 \pm 0,05 \mathrm{~g} /$ recipiente) e o tempo de exposição da madeira ao inseto (30 dias), não foi observado o consumo total dos corpos de prova confeccionados com madeira de P. elliottii.
Segundo Paes et al. (1998), a madeira de Pinus sp. é considerada de baixa resistência, sendo indicada como padrão de comparação para avaliar a resistência natural de madeiras ao ataque de cupins, sendo esperado que tal madeira seja mais avidamente atacada em comparação com a madeira de eucalipto.

\section{CONCLUSÃO}

A perda de massa da madeira de Pinus elliottii foi superior à do Eucalyptus urophylla e Corymbia citriodora, indicando sua maior susceptibilidade à ação de Coptotermes gestroi.

A madeira de E. urophylla e C. citriodora promove maior mortalidade em Coptotermes gestroi, em relação à madeira de Pinus elliottii.

A madeira de Eucalyptus urophylla e de Corymbia citriodora é mais resistente à ação de Coptotermes gestroi, enquanto a de Pinus elliottii é mais susceptível.

A madeira de Eucalyptus urophylla e de Corymbia citriodora sofre menos desgaste por Coptotermes gestroi do que a madeira de Pinus elliottii.

\section{STATUS DA SUBMISSÃO}

Recebido: $20 / 11 / 2010$

Aceito: 17/02/2011

Resumo publicado online: 01/03/2011

Artigo completo publicado: 31/03/2011

\section{AUTOR (ES) PARA CORRESPONDÊNCIA}

\section{Rodrigo Bastos Rodrigues}

Programa de Pós-Graduação em

Ciências Ambientais e Florestais,

Universidade Federal Rural do Rio de Janeiro -

UFRRJ, BR- 465, Km 7, CEP 23890-000,

Seropédica, RJ, Brasil

e-mail: rodrigofloresta2003@yahoo.com.br

\section{Edvá Oliveira Brito}

Departamento de Produtos Florestais, Universidade Federal Rural do Rio de Janeiro - UFRRJ, BR- 465, Km 7, CEP 23890-000, Seropédica, RJ, Brasil

e-mail:, edvabrito@yahoo.com.br 


\section{REFERENNCIAS}

Abreu RLS, Silva KES. Resistência natural de dez espécies madeireiras da Amazônia ao ataque de Nasutitermes macrocephalus (Silvestri) e N. surinamensis (Holmgren) (Isoptera: Termitidae). Revista Árvore 2000; 24(2):229-234.

American Society for Testing and Materials - ASTM. ASTM D-1413. Standard test method for wood preservatives by laboratory soil-block cultures. Philadelphia: ASTM Standards; 1994. vol. 0410, p. 119-21. Annual book.

American Society for Testing and Materials - ASTM. ASTM D-2017. Standard test method of accelerated laboratory test of naturak decay resistance of wood. ASTM Standards; 1994. vol. 0410, p.324-28. Annual book.

American Society for Testing and Materials - ASTM. ASTM D-3345. Standard test method for laboratory evaluation of wood and other cellulosic materials for resistance to termites. ASTM Standards; 1994. vol. 0410, p.119-21. Annual book.

Brito EO. Curso de Engenharia Florestal. Seropédica: Universidade Federal Rural do Rio de Janeiro - UFRRJ; 2011. Comunicação pessoal.

Foelkel E. A madeira dos pinus e sua susceptibilidade ao ataque por cupins-de-madeira-seca e por cupins subterrâneos [cited 2011 jan. 23]. Available from: http:// www.celso-foelkel.com.br/pinus_05.html\#quatorze.

Gomes JI, Silva EMA, Melo ATS. Durabilidade de 15 espécies de madeiras amazônicas em contato com o solo em ambiente sombreado. Belém - PA: EMBRAPA; 2005. Comunicado técnico, 148.

Hunt GM, Garratt GA. Wood preservation. $3^{\text {rd }}$ ed. New York: Mc Graw Hill; 1967.

Lima TG, Silva MDD, Lelles JG, Della Lucia RM. Ensaio de Apodrecimento Acelerado das Madeiras de Eucaliptus microcorys e de 15 Eucaliptus pilularis, pg 42. Floresta e Ambiente 1997; 4:154.

Oliveira APR. Resistência natural da madeira de Corymbia citriodora, ao ataque de cupins subterrâneo, em teste de laboratório [monografia]. Goiás: Fundação Integrada Municipal de Ensino Superior; 2006.
Oliveira JTS. Caracterização da madeira de eucalipto para a construção civil [tese]. São Paulo: Universidade de São Paulo, São Paulo; 1997.

Paes JB, Morais VM, Farias Sobrinho DW, Bakke OA. Resistência natural de nove madeiras do semiárido brasileiro a cupins subterrâneos, em ensaio de laboratório. Cerne 2003; 9(1):36-47.

Paes JB, Morais VM, Lima CR. Resistência natural de nove espécies de madeiras do semi-árido brasileiro a fungos xilófagos em condições de laboratório. Revista Árvore 2004; 28(2):275-282. http://dx.doi.org/10.1590/ S0100-67622004000200014

Paes JB, Vital BR, Lucia TMCD, Lucia RMD. Eficiência do creosoto vegetal contra cupins subterrâneos em testes de laboratório. Revista Árvore 1998; 22(1):89-98.

Paes JB, Vital BR. Resistência natural da madeira de cinco espécies de eucalipto a cupins subterrâneos em testes de laboratório. Revista Árvore 2000; 24(1):1-6.

Paes JB. Efeito da purificação e do enriquecimento do creosoto vegetal em suas propriedades preservativas [tese]. Universidade Federal de Viçosa; 1997.

Paes JB. Resistência de nove painéis a base de madeira a cupins subterrâneos em ensaio de preferência alimentar [CD-ROM]. In: Anais do VIII Encontro Brasileiro em Madeiras e em Estruturas de Madeira; 2002; Uberlândia: UFU. 2002. http://dx.doi.org/10.1590/ S0100-67622004000200014

Panshin AJ, Zeeuw C. Text book of wood technology. $4^{\text {th }}$ ed. New York: Mc Graw Hill; 1980.

Pêgas MRA. Resistência natural de nove espécies de madeiras ao ataque de Coptotermes gestroi (Wasmann, 1896) (Isoptera: Rhinotermitidae) [monografia]. Seropédica, RJ: UFRRJ; 2007.

Richardson BA. Wood preservation. $2^{\text {nd }}$ ed. London: E \& FN SPON; 1993.

Santos MN, Teixeira MLF, Pereira MB, Menezes EB. Avaliação de Estacas de Pinus sp. Como Isca-Armadilha em Diversos Períodos de Exposição a Cupins Subterrâneos. Floresta 2010; 40(1): 29-36.

Supriana N. Notes the resistance of tropical wood against termites. Stockholm: The International Research Group on Wood Preservation; 1985. Doc. IRG /WP/ 1249.

Zobel B. Silvicultural effects on wood properties. IPEF International 1992; 2:31-38. 